\title{
Proteolipid Promoter Activity Distinguishes Two Populations of NG2-Positive Cells throughout Neonatal Cortical Development
}

\author{
Barbara S. Mallon, H. Elizabeth Shick, Grahame J. Kidd, and Wendy B. Macklin \\ Department of Neurosciences, Lerner Research Institute, Cleveland Clinic Foundation, Cleveland, Ohio 44120
}

Transgenic mice expressing enhanced green fluorescent protein (EGFP) driven by the mouse myelin proteolipid protein (PLP) gene promoter have been developed to investigate cells in the oligodendrocyte lineage. Transgene expression is consistent with the developmental expression of PLP, with cells at all stages of oligodendrocyte differentiation clearly visualized. These animals were analyzed to establish the time course of oligodendrocyte progenitor migration, proliferation, and differentiation in neonatal cortex. In these animals, two populations of NG2 proteoglycan-positive oligodendrocyte progenitor cells were identified that exist in postnatal subventricular zone and cortex. These two populations are distinguished by the presence or absence of PLP gene expression. Thus, PLP gene expression defines a subpopulation of NG2-positive cells from very early postnatal ages, which migrates toward the pial surface and proliferates in situ. EGFP ${ }^{+} / \mathrm{NG}^{+}$cells are present in the cortex from postnatal day 1 , and they remain in the cortex as undifferentiated oligodendrocyte progenitors for up to 3 weeks before myelination begins. These data could be explained by the presence of an important inhibitor of oligodendrocyte differentiation in the cortex during this period, which is downregulated in a region-specific manner to allow myelination. On the other hand, it is possible that oligodendrocyte progenitor cells remain undifferentiated in cortex until an essential signal is produced in situ to induce differentiation.

Key words: oligodendroglia; myelin; progenitor cells; enhanced green fluorescent protein; proteolipid protein; NG2 proteoglycan
The current studies use transgenic mice overexpressing enhanced green fluorescent protein (EGFP) to investigate the differentiation program of cells in the oligodendrocyte lineage. Oligodendrocytes are terminally differentiated cells that produce CNS myelin, which is essential for normal neuronal function. Studies on oligodendrocyte progenitor cell origins in embryonic telencephalon suggest that oligodendrocytes are generated from progenitors present initially in ventral locations, which then migrate to dorsal positions (Timsit et al., 1995; Birling and Price, 1998; Nery et al., 2001; Olivier et al., 2001). Control of migration and differentiation of oligodendrocyte progenitors throughout the neonatal cortex from the subventricular zone (SVZ) is an important, relatively unanswered question that we address in this report.

In embryos, oligodendrocyte progenitors express a series of markers, including the platelet-derived growth factor receptor $\alpha$ $(\operatorname{PDGFR} \alpha)$ (Pringle et al., 1992) and the myelin proteolipid (PLP)/DM20 protein gene (Ikenaka et al., 1992; Timsit et al., 1992a). PLP and DM20 proteins are differentially expressed in cells in the oligodendrocyte lineage, and it has been suggested that they may have different roles in the cell. PLP is most highly expressed at the peak of myelination and is thought to play a role in compact myelin, whereas DM20 mRNA is expressed in the

Received Aug. 28, 2001; revised Oct. 16, 2001; accepted Nov. 8, 2001.

This work was supported by National Institutes of Health Grant NS25304. We thank Valerie Stewart and Anamika Gavhane of the Lerner Research Institute Transgenic Core Facility for assistance with the animals, Dr. Judy Drazba of the Lerner Research Institute Imaging Core for help with the low-magnification fluorescence imaging, and Drs. Robert Miller and Bruce Trapp for helpful discussions.

Correspondence should be addressed to Wendy B. Macklin, Department of Neurosciences, NC30, Cleveland Clinic Foundation, 9500 Euclid Avenue, Cleveland, OH 44195. Email: mackliw@ccf.org.

Copyright (C) 2002 Society for Neuroscience $\quad 0270-6474 / 02 / 220876-10 \$ 15.00 / 0$ mouse embryo from at least embryonic day 9.5 (E9.5), as seen by in situ hybridization and by reverse transcription (RT)-PCR (Ikenaka et al., 1992; Timsit et al., 1992a). Thus, DM20 in particular appears likely to play a role in cells other than actively myelinating cells. Characterizing the pattern and localization of $p l p$ gene expression should prove useful in answering questions of oligodendrogenesis and differentiation, and the current transgenic studies provide important insight into these questions.

Postnatally, the immediate in vivo precursor of the oligodendrocyte is a cell expressing both NG2 chondroitin sulfate proteoglycan and $\operatorname{PDGFR} \alpha$. This $\mathrm{NG} 2^{+} / \operatorname{PDGFR} \alpha^{+}$cell, which has only rarely been shown to express the PLP gene (Trapp et al., 1997), differentiates to a premyelinating cell, which contacts and then myelinates axons (Trapp et al., 1997). However, many NG2 ${ }^{+}$ cells are found in the adult brain. This abundant population of cells is of interest with respect to its potential for remyelination in such diseases as multiple sclerosis and its proposed alternative roles in response to injury and/or homeostasis at the node of Ranvier or synapses (Nishiyama et al., 1999; Levine et al., 2001).

We generated transgenic mice using a construct in which the PLP promoter regulatory elements and the PLP 3' untranslated region (UTR) were ligated to EGFP. The expression of EGFP in these mice is extremely high, relative to earlier transgenic mice (Fuss et al., 2000), and can be easily detected in embryonic and postnatal cells at all stages of the oligodendrocyte lineage. In short, these mice provide an invaluable tool for many studies relating to $p l p$ gene expression and oligodendrocyte development. Our data suggest that, from very early stages of development, there may in fact be two populations of NG2-positive oligodendrocyte progenitor cells. We hypothesize that only the PLPexpressing population may become myelinating oligodendrocytes. The other population may differentiate to become the NG2- 
positive cells found in adult brain, which have been proposed to function in an independent neuromodulatory role in the CNS (Bergles et al., 2000), because they contact nodes of Ranvier (Butt and Berry, 2000) and receive synaptic signals from axons (Bergles et al., 2000).

\section{MATERIALS AND METHODS}

Generation of the transgenic construct. pEGFP-N1 (Clontech, Palo Alto, CA) was digested with SmaI and NotI restriction enzymes to excise EGFP, which was cloned into the modified pNEB193 vector (New England Biolabs, Beverly, MA), described by Fuss et al. (2000). The 3' UTR of PLP was excised from a mouse PLP genomic clone (MSDEx7) with BanI/XhoI, blunt ended, and cloned into the SmaI site of pBluescript (Stratagene, La Jolla, CA). The 3' UTR was excised from pBluescript with $X b a \mathrm{I} / A p a \mathrm{I}$, cloned into pcDNA3 (Invitrogen, Carlsbad, CA), from which it was excised as an $X h o I$ fragment and ligated to the $3^{\prime}$ end of the EGFP fragment in the modified pNEB193 vector. The EGFP-3' UTR fragment was then excised with $A s c I$ and $P a c I$ and cloned into the PLP promoter construct first described by Wight et al. (1993) and modified by Fuss et al. (2000). The final DNA fragment, excised with ApaI/PmeI, was injected into pronuclei of B6/CBA fertilized oocytes that were transferred to the oviducts of pseudopregnant mice. Resulting pups were analyzed by Southern blotting (Sambrook et al., 1989) to identify transgenic founders for these PLP-EGFP lines. Genomic DNA from tail biopsies was isolated by standard protocols (Sambrook et al., 1989), and $5 \mu \mathrm{g}$ were used for analysis.

Analysis of EGFP expression in fixed tissue. For analysis of embryos, the day of vaginal plug detection was defined E0.5. At given stages, the dam was perfused with $4 \%$ paraformaldehyde in PBS. Embryos were removed from the uterus, dissected from the placenta, and post-fixed by immersion in $2 \%$ paraformaldehyde in PBS at $4^{\circ} \mathrm{C}$. Young embryos (E10.5-E12.5) were post-fixed for 2-4 hr, and older embryos (E14.5-E18.5) were post-fixed overnight. For sectioning, embryos were embedded in OCT medium and frozen at $-70^{\circ} \mathrm{C}$, and sections $(10 \mu \mathrm{m})$ were cut on a Leica (Wetzlar, Germany) Jung Frigocut $2800 \mathrm{~N}$ cryostat. Postnatal animals were perfused as above, and tissue was post-fixed in $4 \%$ paraformaldehyde in PBS overnight at $4^{\circ} \mathrm{C}$. The brain was removed, post-fixed overnight, and cryoprotected. Coronal sections $(30 \mu \mathrm{m})$ of brain, posterior to the olfactory bulbs and anterior to the ventral hippocampal commissure, were cut under ice-cold PBS on a Leica VT1000S Vibratome and stored at $4^{\circ} \mathrm{C}$ in PBS. In some cases, embryonic brain was dissected before post-fixation for vibratome sectioning. All sections were mounted in Vectashield (Vector Laboratories, Burlingame, CA) for microscopy.

Immunostaining. Free-floating sections were treated with PBS containing 3\% normal goat serum (NGS) and 1\% Triton X-100 for $1 \mathrm{hr}$ at room temperature. Sections were incubated overnight at $4^{\circ} \mathrm{C}$ with NG2 antibody (1:4000; gift from William Stallcup, The Burnham Institute, La Jolla, CA) or PLP/DM20 antibody (clone AA3, 1:100; gift from Steven Pfeiffer, University of Connecticut Health Science Center, Farmington, CT) in PBS containing $1 \%$ NGS $-0.01 \%$ Triton X-100. Sections were washed three times in PBS and incubated with Texas Red- or rhodamineconjugated secondary antibody for $1 \mathrm{hr}$ at room temperature before washing and mounting in Vectashield.

Bromodeoxyuridine treatment and detection. Animals at postnatal day 1 (P1), P4, P6, and P10 were injected once with bromodeoxyuridine (BrdU) $(100 \mu \mathrm{g} / \mathrm{gm}$ body weight) in PBS and killed at 4 or $8 \mathrm{hr}$ after injection. After perfusion, post-fixation, and sectioning as above, the tissue was treated with $2 \mathrm{~N} \mathrm{HCl}$ for $10 \mathrm{~min}$ and $0.1 \mathrm{M}$ sodium borate for $10 \mathrm{~min}$ and washed three times in PBS before the 3\%NGS-1\% Triton X-100 incubation described above. Sections were incubated with 1:1000 BrdU rat monoclonal antibody (Harlan Sera-Lab Limited, Loughborough, UK) overnight as above and were subsequently incubated with a biotinylated anti-rat secondary antibody (Vector Laboratories) in $1 \%$ NGS-0.01\% Triton X-100 before final incubation with 1:5000 Cy5labeled streptavidin (Jackson ImmunoResearch, West Grove, PA) in 1\% NGS- $-0.01 \%$ Triton X-100. The total number of BrdU-labeled cortical cells was quantified using confocal imaging techniques in sections encompassing the entire medial and lateral cortex in each section. Thus, 40 separate, single-slice $40 \times$ images were quantified per section. Sections from different animals were all from the same region, including cingulate cortex, motor cortex, and all lateral cortex up to the piriform cortex. The number of BrdU-positive cells was quantified, and cells were classified as
$\mathrm{EGFP}^{+} / \mathrm{NG}^{-}{ }^{-}, \mathrm{EGFP}^{+} / \mathrm{NG}^{+}$, , or $\mathrm{EGFP}^{-} / \mathrm{NG}^{-}{ }^{-}$. $\mathrm{No} \mathrm{EGFP}^{+} / \mathrm{NG}^{-} /$ $\mathrm{BrdU}^{+}$cells were observed. Sections were quantified from P1, P4, P6, and P10 animals, labeled for either 4 or $8 \mathrm{hr}$. Similar distributions were noted for samples labeled either 4 or $8 \mathrm{hr}$. Data are presented for sections taken from animals labeled with BrdU for $4 \mathrm{hr}$.

$R T-P C R$. Total RNA was extracted from E16 brain and from cortex of P1, P5, and P10 animals according to the method of Chomczynski and Sacchi (1987). Five micrograms were reverse transcribed with Superscript II (Invitrogen) in the presence of random primers according to the instructions on the manufacturer. The resulting cDNA, or RNA that had not been subjected to reverse transcription, was amplified by PCR using primers designed to the start and stop codons of PLP (5'AGTCAGAGTGCCAAAGACATGG-3' and 5'-TCAGAACTTGGTGCCTCG3-', respectively) as described by Timsit et al. (1992b). Amplification was performed in a PTC-200 thermal cycler (MJ Research, Waltham, MA), and conditions used were as described previously (Timsit et al., 1992b). Amplified products were separated on a $2 \%$ agarose gel containing ethidium bromide.

\section{RESULTS}

\section{Generation of PLP-EGFP mice}

To detect stages of the oligodendrocyte lineage in embryos and brain not apparent by other methods, a transgenic construct was generated with a proven PLP promoter (Wight et al., 1993; Spassky et al., 1998; Fuss et al., 2000) in which EGFP was fused to the 3' UTR of PLP. Three founder lines (EGFP3, EGFP5, and EGFP10) were bred to homozygosity and studied for both transgene and myelin gene expression. All lines expressed normal levels of myelin genes but had differing levels of transgene expression (data not shown). The timing and localization of EGFP expression were comparable in all three lines, in both embryos and postnatal mice; the primary difference among these lines was the fluorescence intensity. Data are presented from all three lines.

\section{EGFP expression in postnatal PLP-EGFP animals}

Analysis of P21 brain showed vivid fluorescence in highly myelinated areas, such as corpus callosum, caudate putamen, anterior commissure, and the lateral olfactory tract (Fig. 1 $A, B$ ). These areas are expected to have a large proportion of fully differentiated oligodendrocytes in which PLP expression is high. In addition, cell bodies were easily detectable throughout the cortex. White matter tracts of the cerebellum also showed high expression of EGFP with lower expression in other layers of the cerebellum (Fig. 1B). A cross section of P30 spinal cord showed cell bodies in apparently comparable number in both the gray and white matter, with additional, more diffuse staining in the white matter tracts of myelinated fibers (Fig. 1C).

PLP is a major component of CNS myelin but is also found in Schwann cells of the peripheral nervous system (PNS). EGFP expression was quite strong in sciatic nerve at all ages (Fig. 1D) and was most apparent in cytoplasmic domains in which myelin is not compacted, such as the paranode (arrow), the cell body (arrowhead), and Schmidt-Lantermann incisures (data not shown). It was also present in nonmyelinating cells, such as satellite cells in the sympathetic ganglia (data not shown).

\section{EGFP expression in PLP-EGFP embryos}

EGFP-positive cells were observed in embryonic tissue from at least E10.5 (Fig. 2A). Fluorescence was noted in the basal plate of the diencephalon of the brain, although as expected from previous studies (Timsit et al., 1992a), stronger signal was present in the dorsal root ganglia (arrow) of the PNS. By E14.5, fluorescence was intense around the olfactory lobe (olf), in distinct regions of 

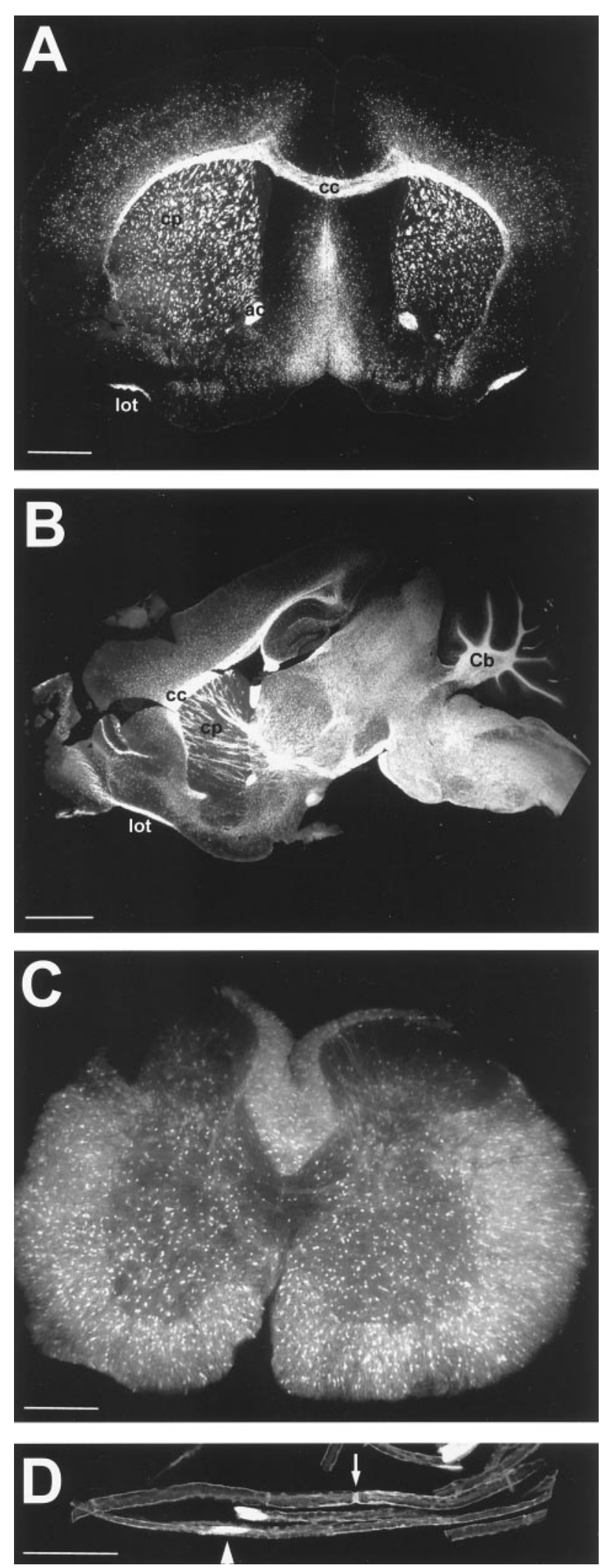

Figure 1. Overview of EGFP expression in postnatal nervous system. $A$, Coronal section of P21 brain (EGFP10) showing vivid fluorescence in white matter tracts, including corpus callosum $(c c)$, caudate putamen $(c p)$, anterior commissure $(a c)$, and the lateral olfactory tract (lot). B, Sagittal section of P21 brain (EGFP5) demonstrating strongest fluorescence in the brainstem and spinal cord, as well as white matter tracts of the cerebellum $(\mathrm{Cb})$. $\mathrm{C}$, Cross section of P30 spinal cord (EGFP3) showing EGFP- the diencephalon $(d i)$, and in the PNS, surrounding the digits of the limbs (Fig. 2B). In more lateral sections, signal was also very apparent in the cerebellar anlage (data not shown). EGFP fluorescence was also seen in the liver, although this was likely autofluorescence.

In the developing spinal cord, it has been demonstrated that oligodendrocyte progenitors originate in the ventral ventricular zone and migrate to populate the dorsal spinal cord (Noll and Miller, 1993). Consistent with these studies, analysis of E14.5 spinal cord, both sagittally and in cross section, demonstrated a predominantly ventral localization of EGFP-positive cells, with a few dorsal cells (Fig. 2B,C).

\section{EGFP expression in cells at multiple stages of the oligodendrocyte lineage at P21}

At the peak of myelination, EGFP was present in many cells in the cortex. Most strikingly, EGFP-positive oligodendrocytes were easily distinguished as mature myelinating oligodendrocytes in P21 cortex, in which they formed multiple parallel processes that were intensely EGFP-positive (Fig. $3 A$ ). In addition, it was seen in cells with several other morphologies, typical of different stages of oligodendrocyte differentiation. Cells with the morphology of premyelinating oligodendrocytes were abundant in P21 cortex (data not shown). These cells are known to express the DM20 protein (Trapp et al., 1997) and would therefore be expected to express the transgene. Cells at even earlier stages of differentiation were noted. Oligodendrocyte progenitors are known to express the chondroitin sulfate proteoglycan NG2, and, even as late as P21-P22, transgene expression could be detected in the cell body of many NG2-positive cells in the cortex (Fig. 3B). This indicated that the PLP promoter was active even in oligodendrocyte progenitors, as would be expected of progenitor cells that are beginning to differentiate into premyelinating oligodendrocytes, and have started to express DM20 protein (Trapp et al., 1997). Thus, at the peak of myelination, cells at multiple stages of differentiation could be identified with this transgene.

\section{EGFP expression in cells at multiple stages of the oligodendrocyte lineage at P1}

In neonatal animals, expression of the transgene could also be seen in cells at several stages of differentiation. Interestingly, these were often observed at developmental stages and locations that were well before significant myelination occurred. To confirm that the transgene was expressed in cells that normally express the plp gene, tissue was stained for PLP/DM20 protein. A distinct band of PLP/DM $20^{+} / \mathrm{EGFP}^{+}$cells was observed in the developing subcortical white matter at P1 (Fig. 3C), although no such cells were found outside the presumptive white matter, indicating early, region-specific differentiation of these cells. The PLP/ $\mathrm{DM}_{20}{ }^{+} / \mathrm{EGFP}^{+}$cells in the subcortical white matter exhibited a range of morphologies (Fig. 3D). Early progenitor cells had green processes and a small area of PLP/DM20 immunostaining on the cell body (Fig. 3D, cell 1). Others had more PLP/DM20 immunoreactivity in the cell body and a few processes stained weakly

positive cell bodies in both gray and white matter. $D$, Teased fibers of 6 month sciatic nerve (EGFP10) showing fluorescence in cell bodies ( $a r$ rowhead) and the paranode (arrow). Scale bars: $A, 1000 \mu \mathrm{m} ; B, 2000 \mu \mathrm{m}$; $C, 500 \mu \mathrm{m} ; D, 25 \mu \mathrm{m}$. 

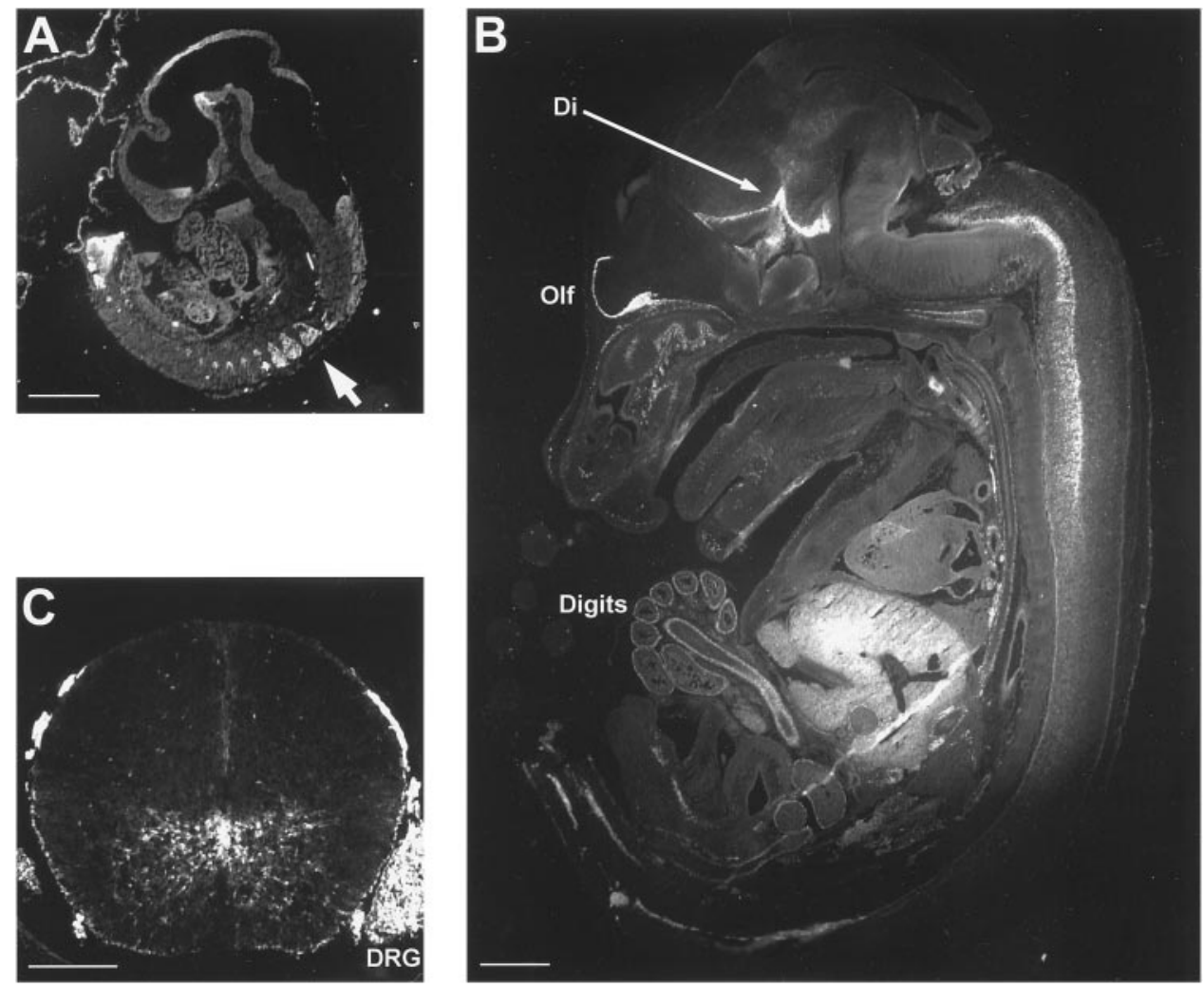

Figure 2. Transgene expression is found in PNS and CNS of embryos from E10.5. $A$, Sagittal cryostat section of E10.5 embryo (EGFP5) showing fluorescence in the basal plate of the diencephalon in the developing brain and the dorsal root ganglia of the PNS (arrow). B, Sagittal cryostat section of E14.5 embryo (EGFP5) showing fluorescence around the olfactory bulb $($ Olf $)$, in the diencephalon $(D i)$, and the spinal cord. Transgene expression is also apparent in PNS structures, including the digits (Digits). $C$, Axial cryostat section of E14.5 embryo spinal cord (EGFP5) demonstrating the mainly ventral localization of transgene expression. Some EGFP-positive cells are visible in the dorsal region. $D R G$, Dorsal root ganglion. Scale bars: $A, B, 1000 \mu \mathrm{m}$; $C, 250 \mu \mathrm{m}$. for PLP/DM20 (Fig. 3D, cell 2), whereas in more advanced cells, the cell body was more intensely green and had a greater number of longer processes that were distinctly stained for PLP/DM20 (Fig. 3D, cells 3, 4). Finally, cells typical of premyelinating oligodendrocytes (Trapp et al., 1997) were present (Fig. 3D, cell 5), which showed the most elaborate array of strongly PLP/DM20stained processes and a cell body that also strongly stained for PLP/DM20.

\section{EGFP expression in NG2-positive cells throughout development}

The distribution of EGFP-positive cells was analyzed relative to the progression of myelinating oligodendrocytes throughout the gray matter of frontal cortex during development (Fig. 4A). As noted above, in the cortex at the youngest postnatal ages, essentially no cells expressed PLP/DM20 protein at levels detectable by standard immunocytochemical methods. However, there were many $\mathrm{EGFP}^{+} / \mathrm{NG}^{+}$cells in the cortex at all ages examined (Figs. $2 A, 5 B$ ). Almost all EGFP-positive cells were NG2positive, except at $\mathrm{P} 1$, in which $\mathrm{EGFP}^{+} / \mathrm{NG}^{-}$cells were noted in some sections. On the other hand, at all time points, a large number of NG2-positive cells were EGFP-negative. Thus, we hypothesize the existence of two populations of NG2-positive cells in postnatal cortex, only one of which expresses the $p l p$ gene. However, despite the presence of a significant NG2-positive cell population in the cortex that expressed the $p l p$ gene at these early ages, we noted no morphological evidence of significant differentiation or change in distribution of these cortical $\mathrm{EGFP}^{+} / \mathrm{NG}^{+}$ cells between $\mathrm{P} 1$ and $\mathrm{P} 10$ (Fig. $4 A$ ). Thus, these cells, which are considered oligodendrocyte progenitor cells, are present in the cortex quite early and remain there in an apparently undifferentiated state for a significant period of time.
Because cells in P1 cortex did not stain for PLP/DM20, it was important to establish that the plp gene was expressed in this tissue. Cortices were dissected free of subcortical white matter from P1, P5, and P10 brains and analyzed by RT-PCR to detect the presence of PLP and DM20 transcripts. Both PLP and DM20 mRNAs were present in all cortical samples, as well as E16.5 whole brain, with DM20 being the more abundant transcript in the E16.5 and P1 cortex samples (Fig. 4B). This confirms that the plp gene is transcribed in these early cortical areas at the time that EGFP is being expressed there, although colocalization of PLP/ DM20 transcripts in EGFP-positive cells has not yet been demonstrated. Most importantly, these cortical samples expressed both EGFP and PLP/DM20 transcripts for at least 2 weeks before active myelination in these areas.

Analysis of early postnatal cortex revealed a significant alteration in EGFP-positive cell morphology between P1 and P6 (Fig. 5). In the cortex of P1 mice, many EGFP-positive cells exhibited an elongated unipolar morphology typical of migrating cells (Fig. $5 A$ ), and these cells expressed NG2 on their extended processes (Fig. 5B). No cells with the premyelinating morphology were noted in cortex at P1. In a similar region of P4 mouse brain, cells with extended processes were still apparent, but some cells appeared in pairs, possibly indicating that they were recently divided cells (Fig. 5C, arrows). By P6, EGFP-positive cells were typically less elongated, and paired cells were more common (Fig. $5 D)$. EGFP-positive cellular processes were more diffuse at this time point. Even at this time point, no cells with the premyelinating morphology were noted in the cortex. These data suggested that, during the early postnatal period, NG2-positive cells migrate radially into cortex and that migration of EGFP-positive oligodendrocyte progenitors through the gray matter may be reduced by approximately P6. At this point, these cells may 
Figure 3. Transgene expression may be detected at all stages of the oligodendrocyte lineage. $A$, EGFP-stained myelinating oligodendrocyte in P21 brain (EGFP5). Note the many parallel processes of myelinated axons. $B, \mathrm{NG} 2 \mathrm{im}$ munostaining of P22 cortex (EGFP10) showing oligodendrocyte progenitor cells. Some NG2-positive cells (Texas Red) are clearly expressing the transgene and are often found in closely apposed pairs or doublets. $C$, PLP/DM20 (Texas Red) immunostaining of P1 subcortical white matter (EGFP10) showing a clear band of premyelinating oligodendrocytes only in the developing subcortical white matter. $D$, PLP/DM20 (Texas Red) immunostaining of P1 corpus callosum (EGFP10). Cells in an apparent progression of differentiated states are numbered $1-5$, as discussed in Results. Scale bars: $A, B, D, 25 \mu \mathrm{m} ; C$, $50 \mu \mathrm{m}$.
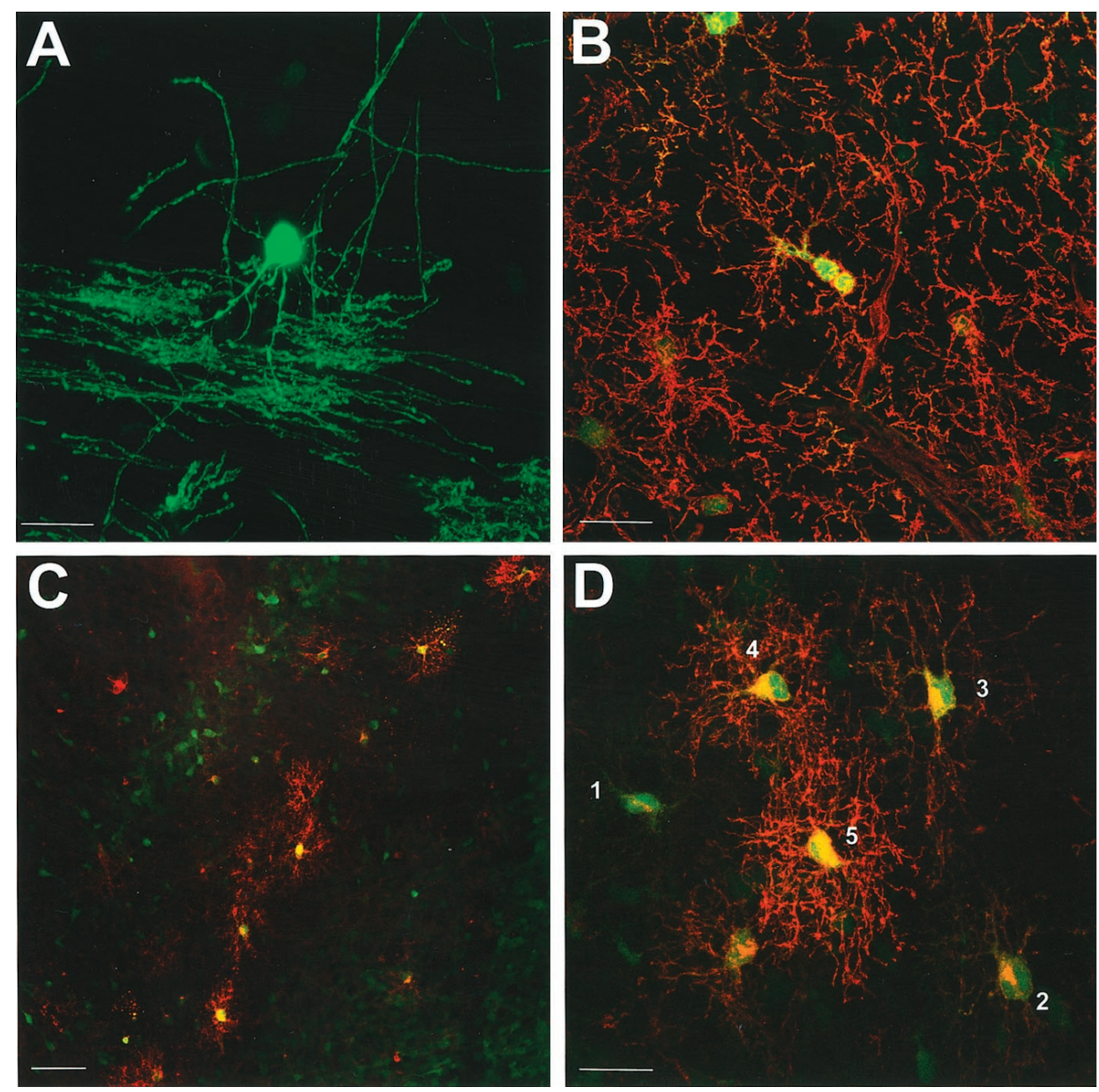

proliferate in situ but demonstrate little evidence of rapid differentiation.

\section{EGFP expression in dividing NG2-positive cells in cortex}

The next important question was whether EGFP expression in NG2-positive cells marked postmitotic cells that were beginning to differentiate into premyelinating and myelinating cells or whether EGFP was expressed in proliferating NG2-positive cells.

To investigate proliferation of these cells and to determine whether the PLP promoter was active before or after cell division, P1, P4, P6, and P10 mice were injected with BrdU (100 $\mu \mathrm{g} / \mathrm{gm}$ body weight) and killed after 4 or $8 \mathrm{hr}$. In P10 animals, $\mathrm{EGFP}^{+} / \mathrm{NG}^{+} / \mathrm{BrdU}^{+}$cells were seen, which appeared to be in the early stages of division. These cells exhibited increased NG2 immunoreactivity in the cell body compared with most NG2positive cells, and they had retracted or unstained processes (Fig. 6A). Redwine and Armstrong (1998) described this morphology in NG2-positive cells in pathological samples in which these cells proliferate. In the current studies, these intensely stained cells were essentially all BrdU positive, and many also expressed EGFP. In addition, by $8 \mathrm{hr}$ after BrdU injection, a number of cells that were in the later stages of division were clearly positive for EGFP, BrdU, and NG2 (Fig. 6B).

Quantification of $\mathrm{EGFP}^{+} / \mathrm{NG}^{+}$cell proliferation in frontal cortex sections revealed that there was a marked increase in the proliferation rate of these cells between $\mathrm{P} 1$ and $\mathrm{P} 4$, followed by a decline toward P10 (Fig. 6C). Thus, at P4, over $20 \%$ of the $\mathrm{EGFP}^{+} / \mathrm{NG}^{+}$cells had undergone DNA synthesis within the past $4 \mathrm{hr}$, suggesting a significant amount of proliferation in this cell population at this age. This is consistent with other studies showing that, between $\mathrm{P} 3$ and $\mathrm{P} 7,30-35 \%$ of all NG2-positive cells incorporate BrdU after a $2 \mathrm{hr}$ pulse (Dawson et al., 2000). It is also consistent with our cell morphology data (Fig. 5), in which cells appeared commonly as pairs from P4 onward. In other studies, it has been observed that the density of NG2-positive cells in the cortex peaks at P7 (Nishiyama et al., 1996), which correlates well with our data, because it suggests that the peak of proliferation may occur near this time.

Most importantly, these BrdU studies demonstrated that the two populations of NG2 cells noted in these animals, i.e., EGFPpositive and EGFP-negative, NG2-positive cells, were separate proliferating cell populations (Fig. 6D). Both populations of $\mathrm{NG}_{2}{ }^{+} / \mathrm{BrdU}^{+}$cells increased with age as a percentage of the total number of dividing cells. Thus, at P1, both populations of proliferating NG2-positive cells together constituted $53 \%$ of the total dividing cells in cortex, and, by P10, they constituted $77 \%$ of the total dividing cells, i.e., by P10, NG2-positive cells became the major proliferating cells in the cortex. These data correlate well with studies demonstrating that $74 \%$ of dividing cells in postnatal brain are NG2 positive (Dawson et al., 2000). This indicates that not only is proliferation a major activity for these cells during this 


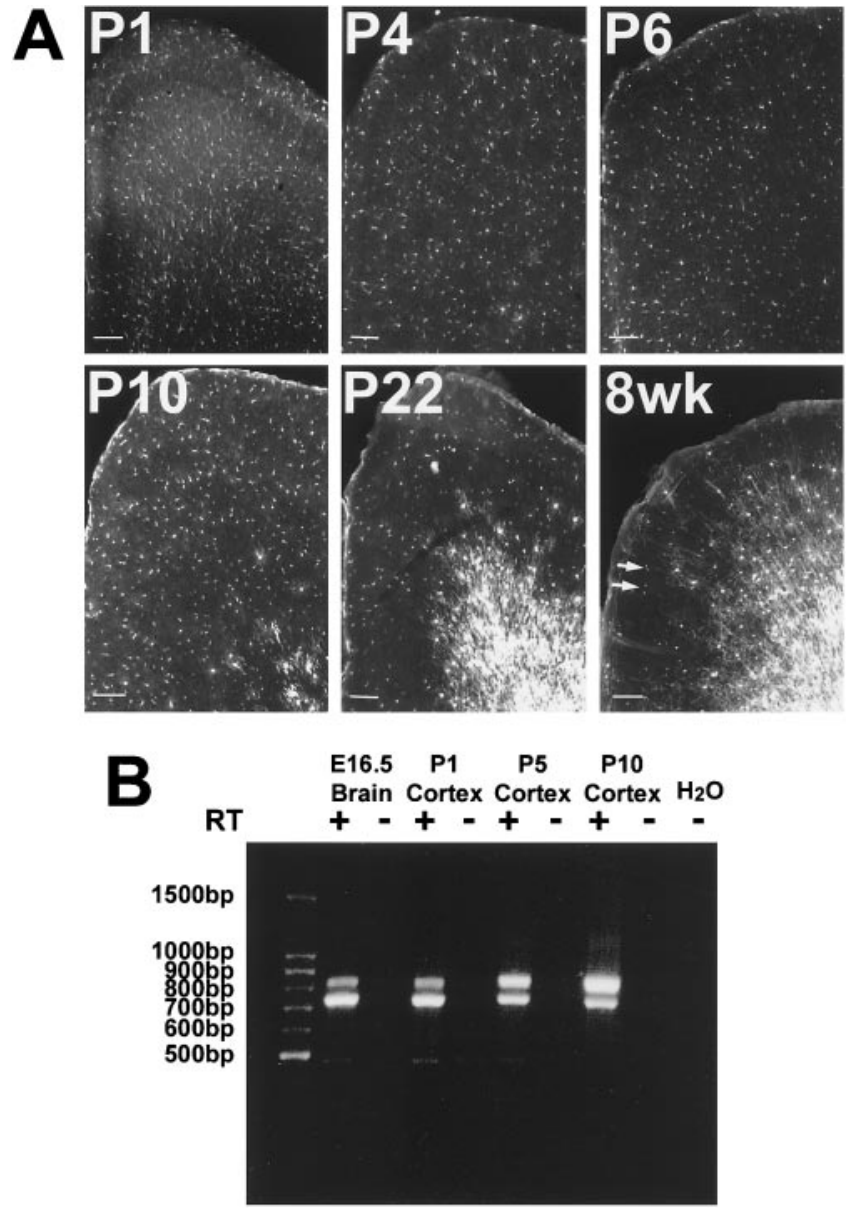

Figure 4. EGFP and $p l p / D M 20$ expression in developing cortex. $A$, Fluorescence imaging of EGFP expression from P1 to 8-week-old animals (EGFP10) in medial frontal sections of cortex (midline is on the left). Intensity of fluorescence increased as the cells became obviously myelinating cells, although cell density did not increase. Arrows in the 8 week sample identify EGFP-positive cells that are also NG2-positive (data not shown). Scale bars, $100 \mu \mathrm{m}$. B, PCR analysis of plp (top band) and DM20 (bottom band) transcripts in cDNA $(+)$ or reverse transcriptase-negative RNA (-) from cortex dissected from P1-P10 animals (EGFP5). DM20 transcripts are the most abundant in the early ages, including E16.5 whole brain.

period, but that NG2-positive cells are the primary type of proliferating cells in early postnatal cortex.

In addition, these studies demonstrate that these proliferating NG2-positive cells consist of two populations, PLP/DM20expressing and nonexpressing NG2-positive cells. The ratio of the dividing $\mathrm{EGFP}^{+} / \mathrm{NG}^{+}{ }^{+}$and $\mathrm{EGFP}^{-} / \mathrm{NG} 2^{+}$cell populations remained relatively constant throughout this period, i.e., the $\mathrm{EGFP}^{+} / \mathrm{NG}^{+}{ }^{+}$cells were slightly less than half of the total dividing NG2-positive cells in the cortex from P1 to P10. This suggests that EGFP expression cannot simply mark postmitotic NG2-positive cells that are moving on into the oligodendrocyte differentiation program to become myelinating oligodendrocytes, but rather that these cells may represent two distinct NG2positive populations that may migrate and proliferate in the same tissue but may serve different functions.

To assess whether this separation of two NG2-positive cell populations existed at an earlier point, cells in the SVZ of P1 mice were analyzed (Fig. 7). Many $\mathrm{EGFP}^{+} / \mathrm{NG}^{+}$cells were noted in the SVZ, along with many $\mathrm{EGFP}^{-} / \mathrm{NG} 2^{+}$cells (arrows). Thus, the existence of two populations of NG2-positive cells was apparent even in the SVZ from the earliest postnatal time.

\section{DISCUSSION}

In this report, we describe transgenic mice in which EGFP, fused to the 3' UTR of PLP, was expressed under the control of the PLP promoter and regulatory elements. Several transgenic mice have been generated previously with variations of this PLP promoter construct (Wight et al., 1993; Spassky et al., 1998; Fuss et al., 2000) to label oligodendrocyte lineage cells in embryonic and postnatal tissue. Because 3' UTR sequences from many mRNAs contain mRNA stabilization and destabilization elements (for review, see Ross, 1995), we generated a new PLP promoter construct fusing the 3' UTR of PLP to a reporter with high signal intensity, EGFP.

The most striking observations from these data are that two populations of NG2-positive cells exist from very early developmental time points, which can be distinguished by $p l p$ promoter activity. Additionally, it appears that the plp-expressing population expresses the $p l p$ gene during migration and cell division as it populates the cortex, but it does not differentiate for days to weeks thereafter.

\section{PLP promoter activity in NG2-positive cells}

The PLP-EGFP transgene significantly enhanced detection and characterization of oligodendrocyte lineage cells in brain and spinal cord, as well as Schwann cells in the PNS and cells in embryonic brain, spinal cord, and PNS. Because of the signal intensity and high degree of transgene expression, cells at many stages of the oligodendrocyte lineage were easily visualized. In early postnatal cortex, many NG2-positive oligodendrocyte progenitor cells were EGFP-positive, i.e., they exhibited plp promoter activity. The proliferation rate of $\mathrm{EGFP}^{+} / \mathrm{NG}^{+}$cells peaked at approximately $\mathrm{P} 4$ in the cortex, and it appeared that the EGFP-positive progenitor cells had completed radial migration into the cortex by at least P6.

NG2 immunoreactivity is first detected on PDGFR $\alpha$-positive cells migrating out of the ventral ventricular zone of rat spinal cord at E14 (Nishiyama et al., 1999). These NG2 ${ }^{+} / \mathrm{PDGFR} \alpha^{+}$ cells have been proposed to differentiate into mature oligodendrocytes because some studies demonstrated a progression of antigen expression from $\mathrm{NG} 2^{+} / 04^{-}$through $\mathrm{NG} 2^{+} / 04^{+}$to $\mathrm{NG} 2{ }^{-} / 04^{+}$/galactosyl cerebroside (GC) ${ }^{+}$(Reynolds and Hardy, 1997). Although many NG2-positive cells differentiate into mature oligodendrocytes, the remaining cells do not disappear but persist into the adult in both gray and white matter, in which they comprise 5-8\% of the total number of cells (Dawson et al., 2000). Several studies have shown that NG2-positive cells, isolated from adult rat cerebellum and optic nerve, are capable of proliferation and differentiation into oligodendrocytes in vitro (Levine et al., 1993; Shi et al., 1998), and it is attractive to hypothesize that they can contribute to remyelination in vivo. On the other hand, other important functions for these adult NG2-positive cells have been proposed that are quite distinct from a role as oligodendrocyte progenitors. Adult NG2-positive cells display a different morphology relative to their younger counterparts (Nishiyama et al., 1999), extending processes along axons to form discrete contacts with axolemma at nodes of Ranvier, perhaps suggesting a role for these cells relating to nodal function (Butt and Berry, 2000). It has also been proposed that adult NG2-positive cells may regulate synaptic growth and plasticity by glutamate signaling at the synapse (Bergles et al., 2000) or that they represent a population of 

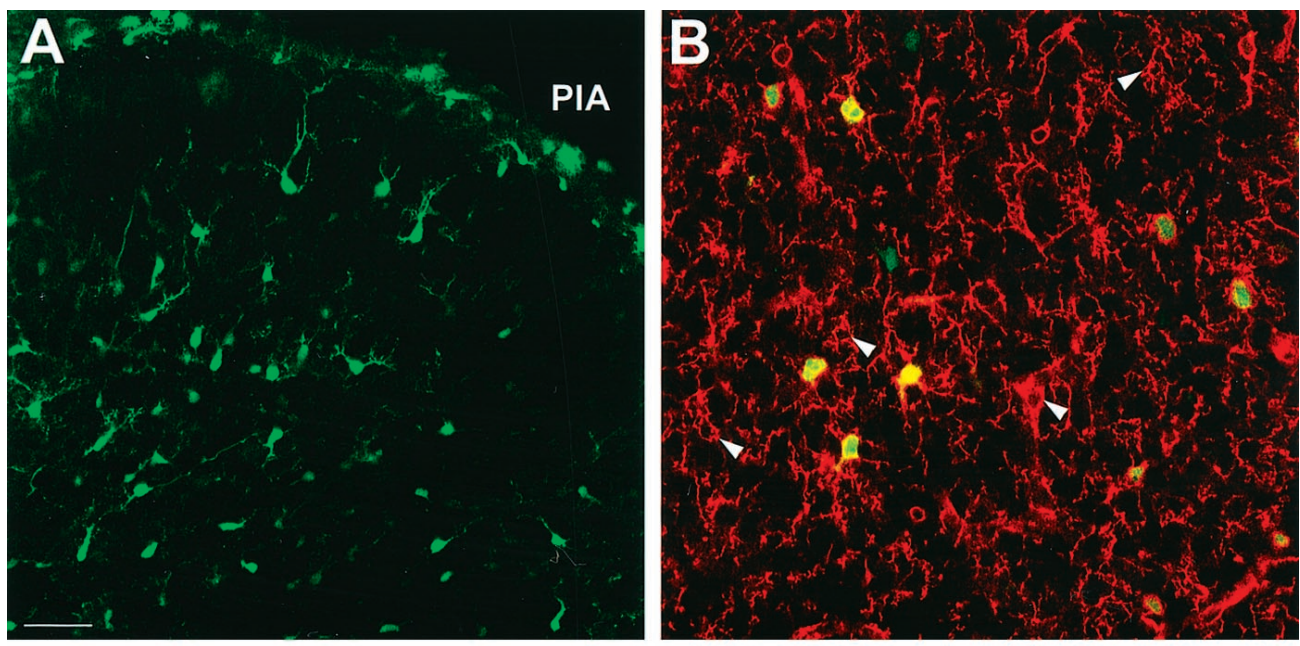

Figure 5. Radial migration through the gray matter is complete by P6. $A$, Many EGFP-positive cells in the outer cortex of $\mathrm{P} 1$ brain appear to have a migratory morphology, with some exhibiting a unipolar morphology (EGFP10). B, NG2 immunostaining of P1 outer cortex (EGFP10) showing that the EGFPpositive cells in P1 cortex also express NG2 (Texas Red). Arrowheads indicate nongreen NG2-positive cells. $C$, By P4, the cells in the outer cortex are beginning to show a few doublets, suggestive of proliferation (see arrows), but some still retain the unipolar shape (EGFP10). $D$, At $\mathrm{P} 6$, the cells populating the outer cortex have lost the unipolar leading process and are often found in pairs (arrowheads; EGFP10). All sections are oriented comparably, with the pial surface at the top right in the image (PIA). Scale bars: $A, C, D, 50 \mu \mathrm{m} ; B, 25 \mu \mathrm{m}$.
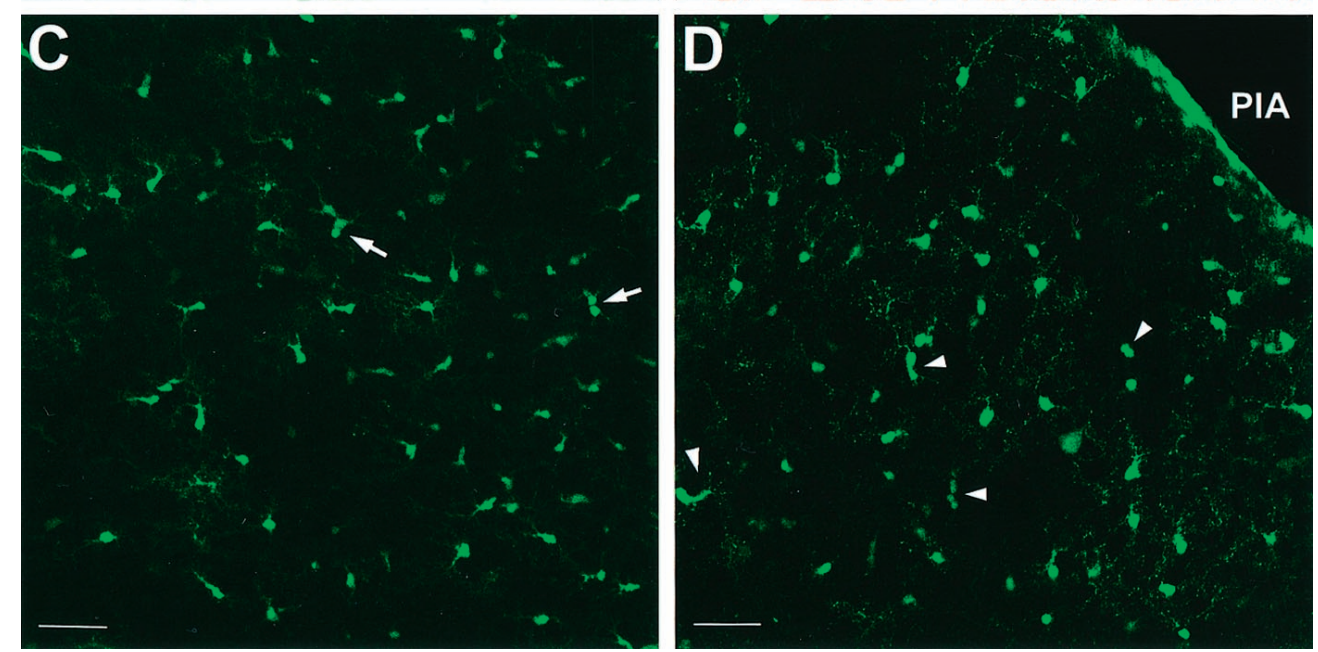

stem-like cells throughout the adult nervous system (Levine et al., 2001).

Given the fact that NG2-positive cells appear to have multiple functions, it has been suggested that there are two populations of adult NG2-positive cells (Keirstead et al., 1998; Nishiyama et al., 1999). In studies in the adult rat spinal cord, two NG2-positive cell populations were termed responsive and nonresponsive based on their activity after GC antibody plus complement-induced demyelination (Keirstead et al., 1998). Other studies have also demonstrated both quiescent and mitotically active oligodendrocyte progenitors in adult brain (Gard and Pfeiffer,1989; Crang and Blakemore, 1997). Although Keirstead et al. (1998) proposed that the responsive population represented oligodendrocyte progenitors, no role for the nonresponsive population was suggested.

Our data would support the existence of two populations of NG2-positive cells and would further suggest that these two populations, distinguished in the current studies by $p l p$ promoter activity, are present not only in the adult but also at very early stages in the normal developing mouse brain. One population, presumably the EGFP-positive cells, might have the potential to differentiate into myelinating oligodendrocytes and function in the adult to remyelinate damaged areas, whereas the $\mathrm{EGFP}^{-}$/ $\mathrm{NG}_{2}{ }^{+}$cells might function at the nodes of Ranvier, synapses, and elsewhere in another function. However, the two populations are apparently closely related, because they seem to have similar morphology, and they migrate and proliferate at the same time in the same regions. Thus, the two populations of NG2-positive cells were seen together in the SVZ, in which they both tended to have an elongated morphology, and they were noted throughout the cortex, in which some had limited processes and others had a multiprocess-bearing morphology.

\section{Oligodendrocyte differentiation}

$\mathrm{EGFP}^{+} / \mathrm{NG}^{+}$cells populate the outer cortex by $\mathrm{P} 1$ and remain there, without apparent differentiation, for weeks before myelination (Fig. $4 A$ ). The early presence of these $\mathrm{EGFP}^{+} / \mathrm{NG}^{+}$ cells, which can even be found in the SVZ, implies that, despite the activity of the $p l p$ promoter, they have not commenced full differentiation. It appears that these $p l p$-expressing cells migrate and proliferate in the cortex but remain undifferentiated for up to 3 weeks. This has been noted in other studies that have shown that oligodendrocyte progenitors populate both cortex and cerebellum long before active myelination, in both the embryo and the early postnatal animal (LeVine and Goldman, 1988; Warrington and Pfeiffer, 1992; Gonye et al., 1994). As noted in the previous studies, it is important to investigate why these cells populate these areas at such early time points and what controls their subsequent differentiation. Thus, it appears that these cells either enter an environment that expresses inhibitors of oligodendrocyte differentiation, in which they cannot differentiate until these inhibitors are downregulated, or they await specific inducing signals to commence myelination. These inhibitory or inducing signals cannot be global but must act locally, because, when 

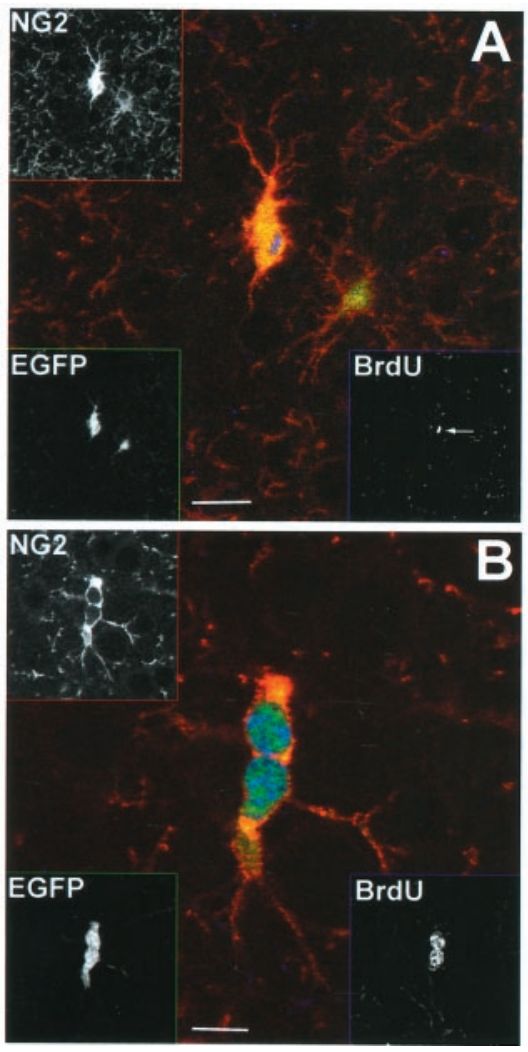
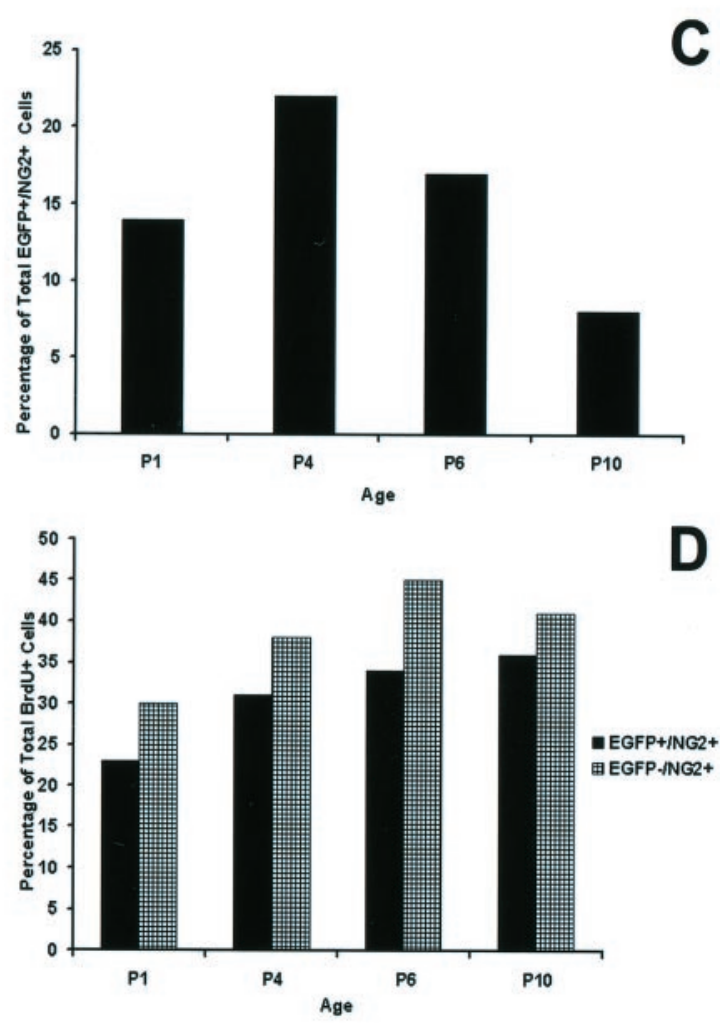

Figure 6. $\mathrm{EGFP}^{+} / \mathrm{NG}^{+}$cells are capable of division. $A$, An oligodendrocyte progenitor cell, positive for the transgene, has retracted processes and upregulated NG2 immunoreactivity (Texas Red, EGFP10). This cell also exhibits a smal intense region of $\mathrm{BrdU}$ staining $(\mathrm{Cy} 5$; see arrow in the spliced image) after $4 \mathrm{hr}$ BrdU labeling, suggesting that this cell may be about to undergo mitosis. Separate channel images are shown in the corners. $B$, A doublet of $\mathrm{EGFP}^{+} / \mathrm{NG}^{+}$ cells that also stain for BrdU after $8 \mathrm{hr}$ labeling, indicating that these cells have recently undergone, or are currently undergoing, mitosis (EGFP10). $C, \mathrm{EGFP}^{+} /$ $\mathrm{NG}^{+} / \mathrm{BrdU}^{+}$cells expressed as a percentage of total $\mathrm{EGFP}^{+} / \mathrm{NG}^{+}{ }^{+}$cells shows that the peak of division of this cell population is at approximately P4. D, Both populations of $\mathrm{NG}_{2}{ }^{+} / \mathrm{BrdU}^{+}$cells are expressed as a percentage of total $\mathrm{BrdU}^{+}$cells, showing that $\mathrm{NG}^{+}$cells become the predominant proliferating cell type after P1 and that the ratio of proliferating EGFP-positive to EGFPnegative/ $\mathrm{NG}_{2}{ }^{+}$cells remains relatively constant with age. Scale bars: $A, 10 \mu \mathrm{m}$; $B, 5 \mu \mathrm{m}$. myelination begins, not all cells in a given area differentiate at the same time (Fig. 4A, P10).

The wave of myelination observed here and in other studies has been proposed to result from local maturation of axons (Hardy and Reynolds,1993; Butt and Berry, 2000), electrical activity (Demerens et al., 1996), or axonal size (Colello et al., 1995). Regardless of the mechanism, if axonal maturation is involved in this signaling, it must be regulated by axonal structure-function at the local level, because there are both ascending and descending axons throughout the cortex, all of which are developing through this period. Thus, axonal growth cones or neuronal cell bodies cannot be involved globally in this regulation.

\section{PLP/DM20 protein expression in neonatal cortex}

It has long been accepted that PLP plays a role in the formation and/or stability of compact myelin and that DM20 may have another role in development or maturation. In fact, it has been shown that both isoforms, but particularly DM20, are required for oligodendrocyte maturation (Nadon and West, 1998). Our studies, as well as others (Spassky et al., 1998), demonstrate that the plp gene is expressed much earlier in the oligodendrocyte lineage than originally thought (Figs. $3 B, 4$ ). What role could these proteins, especially a so-called structural protein like PLP, have in migrating and dividing progenitor cells? PLP has some structural relationship to the tetraspanin protein family, although it is not a member of this family. These proteins have been implicated in signaling to control proliferation, motility, and differentiation in various cell types, in particular through interaction with integrins (Maecker et al., 1997). Recently, we demonstrated that PLP interacts with integrins and is involved in inside-out signaling in cultured oligodendrocytes (T. I. Gudz and W. B. Macklin, unpublished observations). Thus, it is possible that low-level PLP or DM20 protein expression in cells, well before myelination starts,
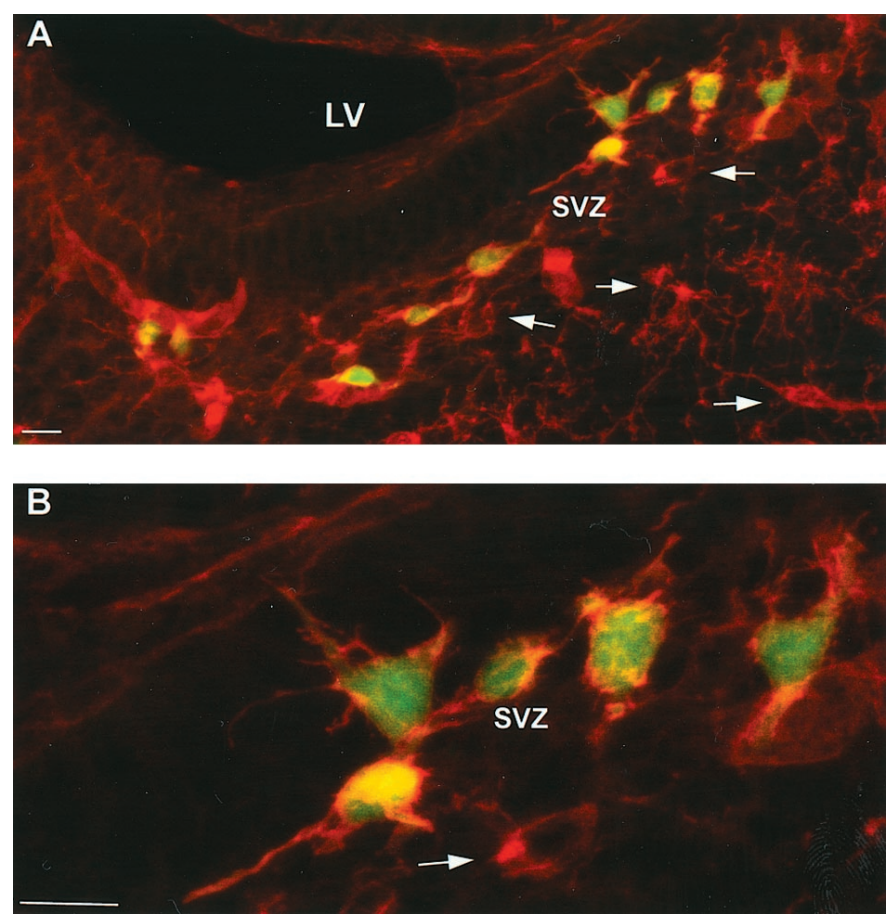

Figure 7. $\mathrm{EGFP}^{+} / \mathrm{NG} 2^{+}$cells are present in the subventricular zone at P1. P1 tissue (EGFP10) was stained for NG2 proteoglycan and imaged for NG2 (Texas Red) and EGFP. $A$, Image of general SVZ area. $L V$, Lateral ventricle. $B$, Higher magnification of cells near the SVZ. Arrows highlight $\mathrm{EGFP}^{-} / \mathrm{NG}^{+}$cells in the SVZ. Scale bars, $10 \mu \mathrm{m}$. 
serves a currently unknown function in proliferation, motility, or differentiation of NG2-positive cells.

In the current and previous studies, it was difficult to doublestain cells for NG2 and PLP/DM20, although some doublestaining was found in a small population of cells (Trapp et al., 1997). This was surprising because cortical tissue contained significant numbers of $\mathrm{EGFP}^{+} / \mathrm{NG}^{+}$cells and the mRNAs for both PLP and DM20 were present (Fig. 4B). As noted previously (LeVine et al., 1990), it is possible that, at early stages of oligodendrocyte development, the PLP/DM20 mRNA is transcribed much earlier than the protein is produced. This would be consistent with other studies in the developing chick embryo, in which PLP/DM20 immunoreactivity was seen in only $20 \%$ of the cells detected by PLP/DM20 in situ hybridization (Perez Villegas et al., 1999) and only in cells that appeared relatively differentiated. It is possible that the protein is present in more immature cells but has a different subcellular localization, such as at the ends of processes, which would not be easy to associate with a given cell body. Support for this hypothesis may be implied from studies on a newly identified PLP-related protein that has an altered $\mathrm{N}$ terminus and different subcellular localization (Bongarzone et al., 1999). Alternatively, the protein produced at these very early stages could be the secreted PLP fragment described by Yamada et al. (1999), which has been proposed to increase the number of oligodendrocytes in mixed glial cultures. Thus, novel PLP-related proteins may be expressed well before active myelination and could have functions quite different from the classic PLP/DM20. In any case, the current studies demonstrate PLP promoter activity in cells at a very early stage in oligodendrocyte development, which is maintained in undifferentiated cells for many days.

In conclusion, we generated transgenic mice in which plp promoter activity can be detected in cells at a very early stage in the lineage. As such, these mice are extremely useful in the study of oligodendrogenesis, migration, and differentiation. We show the existence and persistence of two populations of NG2-positive cells that may play different roles during development. We have also presented evidence that, during development, the control of myelination is provided not by the migration and rapid differentiation of progenitor cells in a specific brain region but rather by signals that become available at a significant time after PLPexpressing progenitor cells arrive at their eventual site of differentiation. Live cell imaging of tissue will likely provide invaluable information regarding the movement of oligodendrocyte precursors and their eventual differentiation.

\section{REFERENCES}

Bergles DE, Roberts JD, Somogyi P, Jahr CE (2000) Glutamatergic synapses on oligodendrocyte precursor cells in the hippocampus. Nature 405:187-191.

Birling MC, Price J (1998) A study of the potential of the embryonic rat telencephalon to generate oligodendrocytes. Dev Biol 193:100-113.

Bongarzone ER, Campagnoni CW, Kampf K, Jacobs EC, Handley VW, Schonmann V, Campagnoni AT (1999) Identification of a new exon in the myelin proteolipid protein gene encoding novel protein isoforms that are restricted to the somata of oligodendrocytes and neurons. J Neurosci 19:8349-8357.

Butt AM, Berry M (2000) Oligodendrocytes, the control of myelination in vivo: new insights from the rat anterior medullary velum. J Neurosci Res 59:477-488.

Chomczynski P, Sacchi N (1987) Single-step method of RNA isolation by acid guanidinium thiocyanate- phenol-chloroform extraction. Anal Biochem 162:156-159.

Colello RJ, Devey LR, Imperato E, Pott U (1995) The chronology of oligodendrocyte differentiation in the rat optic nerve: evidence for a signaling step initiating myelination in the CNS. J Neurosci 15:7665-7672.

Crang AJ, Blakemore WF (1997) Attempts to produce astrocyte cultures devoid of oligodendrocyte generating potential by the use of antimitotic treatment reveal the presence of quiescent oligodendrocyte precursors. J Neurosci Res 49:64-71.

Dawson MR, Levine JM, Reynolds R (2000) NG2-expressing cells in the central nervous system: are they oligodendroglial progenitors? J Neurosci Res 61:471-479.

Demerens C, Stankoff B, Logak M, Anglade P, Allinquant B, Couraud F, Zalc B, Lubetzki C (1996) Induction of myelination in the central nervous system by electrical activity. Proc Natl Acad Sci USA 93:9887-9892.

Fuss B, Mallon B, Phan T, Ohlemeyer C, Kirchhoff F, Nishiyama A, Macklin WB (2000) Purification, analysis of in vivo-differentiated oligodendrocytes expressing the green fluorescent protein. Dev Biol 218:259-274.

Gard AL, Pfeiffer SE (1989) Oligodendrocyte progenitors isolated directly from developing telencephalon at a specific phenotype stage: myelinogenic potential in a defined environment. Development 106:119-132.

Gonye GE, Warrington AE, DeVito JA, Pfeiffer SE (1994) Oligodendrocyte precursor quantitation and localization in perinatal brain using a retrospective bioassay. J Neurosci 14:5365-5372.

Hardy R, Reynolds R (1993) Neuron-oligodendroglial interactions during central nervous system development. J Neurosci Res 36:121-126.

Ikenaka K, Kagawa T, Mikoshiba K (1992) Selective expression of DM20 , an alternatively spliced myelin proteolipid protein gene product, in developing nervous system and in nonglial cells. J Neurochem 58:2248-2253.

Keirstead HS, Levine JM, Blakemore WF (1998) Response of the oligodendrocyte progenitor cell population (defined by NG2 labelling) to demyelination of the adult spinal cord. Glia 22:161-170.

Levine JM, Stincone F, Lee YS (1993) Development and differentiation of glial precursor cells in the rat cerebellum. Glia 7:307-321.

Levine JM, Reynolds R, Fawcett JW (2001) The oligodendrocyte precursor cell in health and disease. Trends Neurosci 24:39-47.

LeVine SM, Goldman JE (1988) Spatial and temporal patterns of oligodendrocyte differentiation in rat cerebrum and cerebellum. J Comp Neurol 277:441-455.

LeVine SM, Wong D, Macklin WB (1990) Developmental expression of proteolipid protein and DM20 mRNAs and proteins in the rat brain. Dev Neurosci 12:235-250.

Maecker HT, Todd SC, Levy S (1997) The tetraspanin superfamily: molecular facilitators. FASEB J 11:428-442.

Nadon NL, West M (1998) Myelin proteolipid protein: function in myelin structure is distinct from its role in oligodendrocyte development. Dev Neurosci 20:533-539.

Nery S, Wichterle H, Fishell G (2001) Sonic hedgehog contributes to oligodendrocyte specification in the mammalian forebrain. Development 128:527-540.

Nishiyama A, H X-Lin, Giese N, H C-Heldin, Stallcup WB (1996) Co-localization of NG2 proteoglycan and PDGF $\alpha$ receptor on $02 \mathrm{~A}$ progenitor cells in the developing rat brain. J Neurosci Res 43:299-314.

Nishiyama A, Chang A, Trapp BD (1999) NG2 ${ }^{+}$glial cells: a novel glial cell population in the adult brain. J Neuropathol Exp Neurol 58:1113-1124.

Noll E, Miller RH (1993) Oligodendrocyte precursors originate at the ventral ventricular zone dorsal to the ventral midline region in the embryonic rat spinal cord. J Neurosci Res 118:563-573.

Olivier C, Cobos I, Perez Villegas EM, Spassky N, Zalc B, Martinez S, Thomas JL (2001) Monofocal origin of telencephalic oligodendrocytes in the anterior entopeduncular area of the chick embryo. Development 128:1757-1769.

Perez Villegas EM, Olivier C, Spassky N, Poncet C, Cochard P, Zalc B, Thomas JL, Martinez S (1999) Early specification of oligodendrocytes in the chick embryonic brain. Dev Biol 216:98-113.

Pringle NP, Mudhar HS, Collarini EJ, Richardson WD (1992) PDGF receptors in the rat CNS: during late neurogenesis, PDGF alphareceptor expression appears to be restricted to glial cells of the oligodendrocyte lineage. Development 115:535-551.

Redwine JM, Armstrong RC (1998) In vivo proliferation of oligodendrocyte progenitors expressing PDGFalphaR during early remyelination. J Neurobiol 37:413-428.

Reynolds R, Hardy R (1997) Oligodendroglial progenitors labeled with the $\mathrm{O} 4$ antibody persist in the adult rat cerebral cortex in vivo. J Neurosci Res 47:455-470.

Ross J (1995) mRNA stability in mammalian cells. Microbiol Rev 59:423-450.

Sambrook J, Fritsch EF, Maniatis T (1989) Molecular cloning: a laboratory manual. Cold Spring Harbor, NY: Cold Spring Harbor Laboratory.

Shi J, Marinovich A, Barres BA (1998) Purification and characterization of adult oligodendrocyte precursor cells from the rat optic nerve. J Neurosci 18:4627-4636.

Spassky N, Goujet-Zalc C, Parmantier E, Olivier C, Martinez S, Ivanova A, Ikenaka K, Macklin WB, Cerruti I, Zalc B, Thomas J-L (1998) Multiple restricted origin of oligodendrocytes. J Neurosci 18: 8331-8343. 
Timsit SG, Bally-Cuif L, Colman DR, Zalc B (1992a) DM-20 mRNA is expressed during the embryonic development of the nervous system of the mouse. J Neurochem 58:1172-1175.

Timsit S, Sinoway MP, Levy L, Allinquant B, Stempak J, Staugaitis SM, Colman DR (1992b) The DM20 protein of myelin: intracellular and surface expression patterns in transfectants. J Neurochem 58:1936-1942.

Timsit S, Martinez S, Allinquant B, Peyron F, Puelles L, Zalc B (1995) Oligodendrocytes originate in a restricted zone of the embryonic ventral neural tube defined by DM-20 mRNA expression. J Neurosci 15:1012-1024.

Trapp BD, Nishiyama A, Cheng D, Macklin W (1997) Differentiation and death of premyelinating oligodendrocytes in developing rodent brain. J Cell Biol 137:459-468.

Warrington AE, Pfeiffer SE (1992) Proliferation and differentiation of $\mathrm{O} 4+$ oligodendrocytes in postnatal rat cerebellum: analysis in unfixed tissue slices using anti-glycolipid antibodies. J Neurosci Res 33:338-353.

Wight PA, Duchala CS, Readhead C, Macklin WB (1993) A myelin proteolipid protein-Lacz fusion protein is developmentally regulated and targeted to the myelin membrane in transgenic mice. J Cell Biol 123:443-454.

Yamada M, Ivanova A, Yamaguchi Y, Lees MB, Ikenaka K (1999) Proteolipid protein gene product can be secreted and exhibit biological activity during early development. J Neurosci 19:2143-2151. 